DOI: $10.17516 / 1997-1397-2020-13-1-26-36$

УДК 532.5.013.4

\title{
On the Asymptotic Behavior of the Conjugate Problem Describing a Creeping Axisymmetric Thermocapillary Motion
}

\author{
Victor K. Andreev* \\ Evgeniy P. Magdenko ${ }^{\dagger}$ \\ Institute of Computational Modelling SB RAS \\ Krasnoyarsk, Russian Federation \\ Siberian Federal University \\ Krasnoyarsk, Russian Federation
}

Received 04.03.2019, received in revised form 10.11.2019, accepted 08.12.2019

\begin{abstract}
In this paper the conditions for the law of temperature behavior on a solid cylinder wall describes, under which the solution of a linear conjugate inverse initial-boundary value problem describing a two-layer axisymmetric creeping motion of viscous heat-conducting fluids tends to zero exponentially with increases of time.
\end{abstract}

Keywords:the conjugate nonlinear inverse problem, interface, a crawling motion.

Citation: V.K.Andreev, E.P.Magdenko, On the Asymptotic Behavior of the Conjugate Problem Describing a Creeping Axisymmetric Thermocapillary Motion, J. Sib. Fed. Univ. Math. Phys., 2020, 13(1), 26-36. DOI: 10.17516/1997-1397-2020-13-1-26-36.

\section{Introduction and preliminaries}

In work [1], the linear conjugate inverse initial boundary value problem describing a twolayer creeping motion of viscous heat-conducting fluids in a cylinder with a solid side surface $r=R_{2}=$ const and interface $r=h(t), 0<h(t)<R_{2}$ was considered

$$
\begin{gathered}
v_{1 t}=\nu_{1}\left(v_{1 r r}+\frac{1}{r} v_{1 r}\right)+f_{1}(t), \quad 0<r<R_{1}, \\
v_{2 t}=\nu_{2}\left(v_{2 r r}+\frac{1}{r} v_{2 r}\right)+f_{2}(t), \quad R_{1}<r<R_{2}, \\
v_{1}\left(R_{1}, t\right)=v_{2}\left(R_{1}, t\right), \quad \int_{0}^{R_{1}} r v_{1}(r, t) d r+\int_{R_{1}}^{R_{2}} r v_{2}(r, t) d r=0, \\
\mu_{1} v_{1 r}\left(R_{1}, t\right)-\mu_{2} v_{2 r}\left(R_{1}, t\right)=-2 æ a_{1}\left(R_{1}, t\right), \\
\left|v_{1}(0, t)\right|<\infty, \quad v_{2}\left(R_{2}, t\right)=0, \\
v_{1}(r, 0)=0, \quad v_{2}(r, 0)=0, \\
\rho_{1} f_{1}(t)=\rho_{2} f_{2}(t)-\frac{2 æ a_{1}\left(R_{1}, t\right)}{R_{1}}
\end{gathered}
$$

*andr@icm.krasn.ru

${ }^{\dagger}$ magdenko_evgeniy@icm.krasn.ruｈttps://orcid.org/0000-0003-2354-1152

(C) Siberian Fēeral University. All rights reserved 
and the closed conjugate problem for functions $a_{j}(r, t)$ is described the following equations:

$$
\begin{gathered}
a_{j t}=\chi_{j}\left(a_{j r r}+\frac{1}{r} a_{j r}\right), \\
a_{j}(r, 0)=a_{j}^{0}(r), \quad\left|a_{1}(0, t)\right|<\infty, \\
a_{2}\left(R_{2}, t\right)=\alpha(t), \\
a_{1}\left(R_{1}, t\right)=a_{2}\left(R_{1}, t\right), \quad k_{1} a_{1 r}\left(R_{1}, t\right)=k_{2} a_{2 r}\left(R_{2}, t\right) .
\end{gathered}
$$

The interface is described by the formula

$$
h(t)=R_{1}\left[1+\mathrm{M} h_{1}(t)\right], \quad h_{1}(t)=-\frac{1}{R_{1}} \int_{0}^{t} r v_{1}\left(R_{1}, t\right) d t .
$$

Here $\mathrm{M}=æ a^{1} R_{1}^{3} / \mu_{1} \chi_{1}$ is Marangoni number, $a^{1}=\max _{t \in[0, T]}|\alpha(t)|$. Note that $\mathrm{M} \rightarrow 0$ since the creeping motion considers in this paper.

In paper [1] the priori estimates were obtained for the functions $v_{j}(r, t), a_{j}(r, t), f_{j}(t)$. In this paper, it will be proved that under certain conditions which set for the temperature on the cylinder surface, the solution of the problem (1)-(11) tends to zero exponentially with increasing time.

\section{The behavior of the solution under $t \rightarrow \infty$}

A priori estimates for the function $a_{j}(r, t)$ satisfying the problem (8)-(11) have form [1]

$$
\begin{gathered}
\left|a_{1}(r, t)\right| \leqslant 2\left[\max _{t \in[0, T]}|\alpha(t)|+\frac{1}{\left(R_{1}^{2} k_{2} \rho_{2} c_{\rho_{2}}\right)^{1 / 4}} \max _{t \in[0, T]}\left(A(t) A_{1}(t)\right)^{1 / 4}\right]+\max _{r \in\left[0, R_{1}\right]}\left|a_{1}^{0}(r)\right|, \\
\left|a_{2}(r, t)\right| \leqslant|\alpha(t)|+2\left(\frac{1}{R_{1}^{2} k_{2} \rho_{2} c_{\rho_{2}}} A(t) A_{1}(t)\right)^{1 / 4}
\end{gathered}
$$

where

$$
\begin{gathered}
A(t) \leqslant\left(\sqrt{A_{0}}+\frac{1}{2} \int_{0}^{t} G(\tau) e^{\eta \tau} d \tau\right)^{2} e^{-2 \eta t} \\
A_{1}(t)=k_{1} \int_{0}^{R_{1}} r\left(a_{1 r}^{0}\right)^{2} d r+k_{2} \int_{R_{1}}^{R_{2}} r\left(\bar{a}_{2 r}^{0}\right)^{2} d r+\rho_{2} c_{p_{2}} \int_{0}^{t} \int_{R_{1}}^{R_{2}} r g_{2}(r, t) d r d t .
\end{gathered}
$$

Here $A_{0}$ is value of function $A(t)$ at $t=0$ and

$$
\begin{gathered}
G(t)=\max _{j}\left(\frac{2}{\rho_{j} c_{p_{j}}}\right)^{1 / 2}\left(\int_{R_{1}}^{R_{2}} r g_{2}^{2} d r\right)^{1 / 2}, \\
\bar{a}_{2}(r, t)=a_{2}(r, t)-\frac{\alpha(t)\left(r-R_{1}\right)^{2}}{\left(R_{2}-R_{1}\right)^{2}}, \\
g_{2}(r, t)=\frac{2 \chi_{2} \alpha(t)}{\left(R_{2}-R_{1}\right)^{2}}\left(2-\frac{R_{1}}{r}\right)-\frac{\alpha^{\prime}\left(r-R_{1}\right)^{2}}{\left(R_{2}-R_{1}\right)^{2}} .
\end{gathered}
$$


If the function $\alpha(t)$ and its derivatives $\alpha^{\prime}(t), \alpha^{\prime \prime}(t), \alpha^{\prime \prime \prime}(t)$ are defined for all $t \geqslant 0$, there is a question about the behavior of the problems solutions (1)-(11) at $t \rightarrow \infty$. From the definition of (19) the inequality is valid for the functions $g_{2}(r, t)$

$$
\begin{aligned}
& \int_{R_{1}}^{R_{2}} r g_{2}^{2} d r \leqslant \frac{2}{\left(R_{2}-R_{1}\right)^{4}} \int_{R_{1}}^{R_{2}}\left[4 \chi_{2}^{2}\left(2-\frac{R_{1}}{r}\right)^{2} \alpha^{2}(t)+\right. \\
& \left.+\left(r-R_{1}\right)^{4}\left(\alpha^{\prime}(t)\right)^{2}\right] r d r \leqslant 2 R_{2}\left(R_{2}-R_{1}\right)\left(\alpha^{\prime}(t)\right)^{2}+\frac{32 \chi_{2}^{2} \alpha^{2}(t)}{\left(R_{2}-R_{1}\right)^{3}}
\end{aligned}
$$

(for integrals over $r$, an upper estimate is given but not their exact value, which can be quite cumbersome), so from (17) we have

$$
\begin{aligned}
G(t) \leqslant\left[\max _{j}\left(\frac{2}{\rho_{j} c_{\rho_{j}}}\right)\right]^{1 / 2}\left[2 R_{2}\left(R_{2}-R_{1}\right)\left(\alpha^{\prime}(t)\right)^{2}+\frac{32 \chi_{2}^{2} \alpha^{2}(t)}{\left(R_{2}-R_{1}\right)^{3}}\right]^{1 / 2} \leqslant \\
\quad \leqslant 2\left[\max _{j}\left(\frac{1}{\rho_{j} c_{\rho_{j}}}\right)\right]^{1 / 2}\left[\frac{4 \chi_{2}}{\left(R_{2}-R_{1}\right)^{3 / 2}}|\alpha(t)|+\sqrt{R_{2}\left(R_{2}-R_{1}\right)}\left|\alpha^{\prime}(t)\right|\right] .
\end{aligned}
$$

So from (15) we obtain

$$
\begin{aligned}
A(t) \leqslant\left\{\sqrt{A_{0}}+\left[\max _{j}\left(\frac{1}{\rho_{j} c_{\rho_{j}}}\right)\right]^{1 / 2}\right. & {\left[\frac{4 \chi_{2}}{\left(R_{2}-R_{1}\right)^{3 / 2}} \int_{0}^{t}|\alpha(\tau)| e^{\eta \tau} d \tau+\right.} \\
& \left.\left.+\sqrt{R_{2}\left(R_{2}-R_{1}\right)} \int_{0}^{t}\left|\alpha^{\prime}(\tau)\right| e^{\eta \tau} d \tau\right]\right\}^{2} e^{-2 \eta t} .
\end{aligned}
$$

From (16) and (19) the estimate is valid

$$
\begin{aligned}
\left|A_{1}(t)\right| \leqslant k_{1} & \int_{0}^{R_{1}} r\left(a_{1 r}^{0}\right)^{2} d r+k_{2} \int_{R_{1}}^{R_{2}} r\left(\bar{a}_{2 r}^{0}\right)^{2} d r+ \\
& +\rho_{2} c_{\rho_{2}} R_{2}\left[\frac{4 \chi_{2}}{R_{2}-R_{1}} \int_{0}^{t}|\alpha(\tau)| d \tau+\left(R_{2}-R_{1}\right) \int_{0}^{t}\left|\alpha^{\prime}(\tau)\right| d \tau\right] .
\end{aligned}
$$

We suppose that the following integrals converge

$$
\int_{0}^{\infty}|\alpha(\tau)| e^{\eta \tau} d \tau, \quad \int_{0}^{\infty}\left|\alpha^{\prime}(\tau)\right| e^{\eta \tau} d \tau
$$

then the expression for function modules $|\alpha(\tau)|$ and $\left|\alpha^{\prime}(\tau)\right|$ have the form

$$
|\alpha(\tau)|=\alpha_{1}(t) e^{-\eta \tau}, \quad\left|\alpha^{\prime}(\tau)\right|=\alpha_{2}(t) e^{-\eta \tau}
$$

with non-negative functions $\alpha_{1}(t), \alpha_{2}(t)$, at that $\alpha_{1}(t) \rightarrow 0, \alpha_{2}(t) \rightarrow 0$ at $t \rightarrow \infty$ and the following estimate is valid

$$
\int_{0}^{\infty} \alpha_{k}(\tau) d \tau<\infty, \quad k=1,2
$$

The convergence of integrals

$$
\int_{0}^{\infty}|\alpha(\tau)| d \tau, \quad \int_{0}^{\infty}\left|\alpha^{\prime}(\tau)\right| d \tau
$$


follows from (24), (25), so from (14), (21), (22) we obtain exponential convergence to zero of the function $a_{2}(r, t) \forall r \in\left[R_{1}, R_{2}\right]$ :

$$
\left|a_{2}(r, t)\right| \leqslant \alpha_{1}(t) e^{-\eta t}+2\left(\frac{A_{1}(\infty) D^{2}}{R_{1}^{2} k_{2} \rho_{2} c_{\rho_{2}}}\right)^{1 / 4} e^{-\eta t / 2},
$$

where in the quality $D$ we have designed the value of the expression in curly brackets (21) at $t=\infty$.

For $a_{1}(r, t)$ from the estimate (13)we find

$$
\left|a_{1}(r, t)\right| \leqslant 2\left[\alpha_{1}(t) e^{-\eta t}+\left(\frac{A_{1}(\infty) D^{2}}{R_{1}^{2} k_{2} \rho_{2} c_{\rho_{2}}}\right)^{1 / 4} e^{-\eta t / 2}\right]+\max _{r \in\left[0, R_{1}\right]}\left|a_{1}^{0}(r)\right| \exp \left(-\frac{\chi_{1} \xi_{1} t}{R_{1}}\right),
$$

where $\xi_{1} \approx 2.4048$ is the first roots of equation $J_{0}(\xi)=0$ [2]. So there is

Lemma 2.1. If the functions $\alpha(\tau), \alpha^{\prime}(\tau)$ satisfy conditions (23)-(25), then for the solutions of the initial-boundary value problems (8)-(11) $a_{j}(r, t)$ the following estimates are valid: (26), (27), from which it follows that these functions tend exponentially to zero with increasing time.

The priori estimates for functions $v_{j}(r, t)$ and $f_{j}(t)$ have form [1]

$$
\begin{aligned}
&\left|v_{2}(r, t)\right| \leqslant \frac{2 æ}{\mu_{2}}\left|a_{1}\left(R_{1}, t\right)\right| \max _{r \in\left[R_{1}, R_{2}\right]}\left|P_{4}(r)\right|+\sqrt{\frac{2}{R_{1}}}\left(\frac{2}{\rho_{2} \mu_{2}} H_{2}(t) E(t)\right)^{1 / 4} . \\
&\left|f_{1}(t)\right| \leqslant 2 \nu_{1}\left[\left(\frac{1}{7} R_{1}^{4}+\sum_{n=1}^{\infty}\left|h_{n}^{2}\right|\right)+2 R_{1}^{2} \sum_{n=1}^{\infty}\left(\frac{\left|h_{n}^{1}\right|}{\zeta_{n}^{2}}+\frac{\left|h_{n}^{2}\right|}{R_{1}^{2}}\right)\right] \max _{t \in[0, T]}|g(t)|+ \\
&+\frac{R_{2}^{2}-R_{1}^{2}}{R_{1}^{2}}\left[\frac{2 æ}{\mu_{2}} \max _{t \in[0, T]}\left|a_{1 t}\left(R_{1}, t\right)\right| \max _{r \in\left[R_{1}, R_{2}\right]}\left|P_{4}(r)\right|+\right. \\
&\left.+\sqrt{\frac{2}{R_{1}}} \max _{t \in[0, T]}\left(\frac{2}{\rho_{2} \mu_{2}} H_{3}(t) E_{1}(t)\right)^{1 / 4}\right] . \\
&\left|v_{1}(r, t)\right| \leqslant R_{1} \max _{t \in[0, T]}\left|v_{2}\left(R_{1}, t\right)\right|+\frac{2 R_{1}}{\nu_{1}} \max _{t \in[0, T]}\left|f_{1}(t)\right| \sum_{n=1}^{\infty} \frac{1}{\xi_{n}^{3}\left|J_{1}\left(\xi_{n}\right)\right|}, \\
&\left|f_{2}(t)\right| \leqslant \rho\left|f_{1}(t)\right|+\frac{2 æ}{\rho_{2} R_{1}} \max _{t \in[0, T]}\left|a_{1}\left(\rho_{2} R_{1}, t\right)\right| .
\end{aligned}
$$

Here $\rho=\rho_{1} / \rho_{2}, \xi_{n}$ are the roots of the Bessel function $J_{0}\left(\xi_{n}\right)=0, \zeta_{n}$ are the positive roots of equation $J_{2}(\zeta)=0[3], h_{n}^{1}=\beta_{n}^{1} / \zeta_{n}$ and $h_{n}^{2}=\beta_{n}^{2} / \zeta_{n}$ (where $\beta_{n}^{1}, \beta_{n}^{2}$ are coefficients of Fourier series of functions $-15 R_{1} r$ and $3 R_{1}\left(r^{3}-4 R_{1} r^{2} / 7\right)$ when they are decomposed by function $\left.J_{2}\left(R_{1}^{-1} \zeta_{n} r\right)[1]\right)$. Further we have

$$
P_{4}(r)=\frac{1}{R_{1}^{2}\left(R_{1}-R_{2}\right)}\left(r^{2}-\left(R_{1}+R_{2}\right) r+R_{1} R_{2}\right)\left(r^{2}+C_{1} r+C_{2}\right)
$$

with constants

$$
C_{1}=-\frac{\left(R_{1}+R_{2}\right)\left(2 R_{1}^{2}+2 R_{2}^{2}+R_{1} R_{2}\right)}{\left(R_{2}-R_{1}\right)\left(3 R_{2}+2 R_{1}\right)}, \quad C_{2}=-R_{1} C_{1}
$$

and

$$
E(t) \leqslant\left[\sqrt{E(0)}+\int_{0}^{t} H_{1}(\tau) e^{\delta \tau} d \tau\right]^{2} e^{-2 \delta t}
$$




$$
\begin{gathered}
E(0)=\frac{2 æ^{2} \rho_{2}}{\mu_{2}^{2}}\left(a_{1}^{0}\left(R_{1}\right)\right)^{2} \int_{R_{1}}^{R_{2}} r P_{4}^{2}(r) d r . \\
\left.H_{1}(t)=\left[\sqrt{\frac{\rho_{2}}{2}}\left(\int_{R_{1}}^{R_{2}} r Q_{2}^{2} d r\right)^{1 / 2}+\frac{\Re}{\sqrt{\rho_{1}}} \mid a_{1}\left(R_{1}, t\right)\right]\right] \\
Q_{2}(r, t)=\frac{2 æ}{\mu_{2}}\left[a_{2 t}\left(R_{1}, t\right) P_{4}(r)-\nu_{2}\left(P_{4 r r}+\frac{1}{r} P_{4 r}\right) a_{2}\left(R_{1}, t\right)\right] . \\
H_{2}(t)=\mu_{2} \int_{R_{1}}^{R_{2}} r\left(\bar{v}_{2 r}^{0}\right)^{2} d r+\frac{\rho_{2}}{2} \int_{0}^{t} \int_{R_{1}}^{R_{2}} r Q_{2}^{2}(r, t) d r d t+\frac{æ^{2}}{\rho_{1}} \int_{0}^{t} a_{1}^{2}\left(R_{1}, t\right) d t,
\end{gathered}
$$

Below, in order to determine the behavior of $v_{1}(r, t)$ and $f_{j}(t)$ for large $t$, we need the estimate $\left|a_{2 t}(r, t)\right|$. It was obtained in [1], that

$$
\left|a_{2 t}(r, t)\right| \leqslant\left|\alpha^{\prime}(t)\right|+2\left(\frac{1}{R_{1}^{2} k_{2} \rho_{2} c_{\rho_{2}}} A_{2}(t) A_{3}(t)\right)^{1 / 4}
$$

where

$$
\begin{gathered}
A_{2}(t)=\frac{\rho_{1} c_{\rho_{1}}}{2} \int_{0}^{R_{1}} r a_{1 t}^{2}(r, t) d r+\frac{\rho_{2} c_{\rho_{2}}}{2} \int_{R_{1}}^{R_{2}} r \bar{a}_{2 t}^{2}(r, t) d r \\
A_{20}=A_{2}(0)=\frac{\chi_{1}^{2} \rho_{1} c_{\rho_{1}}}{2} \int_{0}^{R_{1}} r\left(a_{1 r r}^{0}+\frac{1}{r} a_{1 r}^{0}\right)^{2} d r+ \\
+\frac{\rho_{2} c_{\rho_{2}}}{2} \int_{R_{1}}^{R_{2}}\left[\chi_{2}\left(\bar{a}_{2 r r}^{0}+\frac{1}{r} \bar{a}_{2 r}^{0}\right)+\frac{2 \chi_{2} \alpha(0)}{\left(R_{2}-R_{1}\right)^{2}}\left(2-\frac{R_{1}}{r}\right)-\frac{\alpha^{\prime}(0)\left(r-R_{1}\right)^{2}}{\left(R_{2}-R_{1}\right)^{2}}\right]^{2} d r, \\
A_{3}(t)=k_{1} \chi_{1}^{2} \int_{0}^{R_{1}} r\left(a_{1 r r}^{0}+\frac{1}{r} a_{1 r}^{0}\right)^{2} d r+a_{2}^{0}(r)-\frac{\alpha(0)\left(r-R_{1}\right)^{2}}{\left(R_{2}-R_{1}\right)^{2}} ; \\
+k_{2} \int_{R_{1}}^{R_{2}} r\left[\chi_{2}\left(a_{2 r r}^{0}+\frac{1}{r} a_{2 r}^{0}\right)-\frac{\alpha^{\prime}(0)\left(r-R_{1}\right)^{2}}{\left(R_{2}-R_{1}\right)^{2}} d r+\rho_{2} c_{\rho_{2}} \int_{0}^{R_{2}} \int_{R_{1}}^{R_{2}} r g_{3}(r, t) d r d t\right. \\
g_{3}(r, t)=\frac{1}{\left(R_{2}-R_{1}\right)^{2}}\left[2 \chi_{2} \alpha^{\prime}(t)\left(2-\frac{R_{1}}{r}\right)-\alpha^{\prime \prime}(t)\left(r-R_{1}\right)^{2}\right] .
\end{gathered}
$$

Therefore, for $A_{2}(t)$ we obtain inequality (21) with replacement $A_{0}$ by $A_{10}, \alpha(\tau)$ by $\alpha^{\prime}(\tau)$ and $\alpha^{\prime}(\tau)$ by $\alpha^{\prime \prime}(\tau)$. For the function $A_{3}(t)$ inequality form (22) is satisfied with the replacement

$$
\begin{gathered}
\int_{0}^{R_{1}} r\left(a_{1 r}^{0}\right)^{2} d r \quad \text { by } \quad \chi_{1}^{2} \int_{0}^{R_{1}} r\left(a_{1 r r}^{0}+\frac{1}{r} a_{1 r}^{0}\right)^{2} d r \equiv d_{1}, \\
\int_{R_{1}}^{R_{2}} r\left(\bar{a}_{2 r}^{0}\right)^{2} d r \quad \text { by } \int_{R_{1}}^{R_{2}} r\left[\chi_{2}\left(a_{2 r r}^{0}+\frac{1}{r} a_{2 r}^{0}\right)-\frac{\alpha^{\prime}(0)\left(r-R_{1}\right)^{2}}{\left(R_{2}-R_{1}\right)^{2}}\right]^{2} d r \equiv d_{2}
\end{gathered}
$$

and $\alpha(\tau)$ by $\alpha^{\prime}(\tau), \alpha^{\prime}(\tau)$ by $\alpha^{\prime \prime}(\tau)$.

In addition to $(23)-(25)$ we assume the convergence of the integral

$$
\int_{0}^{\infty}\left|\alpha^{\prime \prime}(\tau)\right| e^{\eta \tau} d \tau<\infty
$$


so that there is valid

$$
\left|\alpha^{\prime \prime}(t)\right|=\alpha_{3}(t) e^{-\eta t}, \quad \int_{0}^{\infty} \alpha_{3}(\tau) d \tau<\infty, \quad \alpha_{3}(t) \rightarrow 0 \text { at } t \rightarrow \infty .
$$

Taking into account the above, we find from (14) that

$$
\left|a_{2 t}(r, t)\right| \leqslant \alpha_{2}(t) e^{-\eta t}+2\left(\frac{A_{3}(\infty) D_{1}^{2}}{R_{1}^{2} k_{2} \rho_{2} c_{\rho_{2}}}\right)^{1 / 4} e^{-\eta t / 2},
$$

where

$$
\begin{gathered}
A_{3}(\infty)=k_{1} d_{1}+k_{2} d_{2}+\frac{R_{2} \rho_{2} c_{p_{2}}}{R_{2}-R_{1}}\left[4 \chi_{2} \int_{0}^{\infty}\left|\alpha^{\prime}(\tau)\right|+\left(R_{2}-R_{1}\right)^{2} \int_{0}^{\infty}\left|\alpha^{\prime \prime}(\tau)\right| d \tau\right], \\
D_{1}=\sqrt{A_{10}}+\max _{j}\left(\frac{1}{\rho_{j} c_{p_{j}}}\right)^{1 / 2}\left[\frac{4 \chi_{2}}{\left(R_{2}-R_{1}\right)^{3 / 2}} \int_{0}^{\infty}\left|\alpha^{\prime}(\tau)\right| e^{\eta \tau} d \tau+\right. \\
\left.+\sqrt{R_{2}\left(R_{2}-R_{1}\right)} \int_{0}^{\infty}\left|\alpha^{\prime \prime}(\tau)\right| e^{\eta \tau} d \tau\right] .
\end{gathered}
$$

We turn to inequality for $\left|a_{2 t t}(r, t)\right|[1]$. We have

$$
\left|a_{2 t t}(r, t)\right| \leqslant\left|\alpha^{\prime \prime}(t)\right|+2\left(\frac{1}{R_{1}^{2} k_{2} \rho_{2} c_{\rho_{2}}} A_{4}(t) A_{5}(t)\right)^{1 / 4},
$$

where

$$
\begin{gathered}
A_{4}(t)=\frac{\rho_{1} c_{p_{1}}}{2} \int_{0}^{R_{1}} r a_{1 t t}^{2} d r+\frac{\rho_{2} c_{p_{2}}}{2} \int_{R_{1}}^{R_{2}} r \bar{a}_{2 t t}^{2} d r \\
A_{40}=\frac{\rho_{1} c_{p_{1}}}{2} \int_{0}^{R_{1}} r\left(a_{1 t t}^{0}(r)\right)^{2} d r+\frac{\rho_{2} c_{p_{2}}}{2} \int_{R_{1}}^{R_{2}} r\left(\bar{a}_{2 t t}^{0}(r)\right)^{2} d r .
\end{gathered}
$$

The initial data are found from equations (9) and replacement of (18):

$$
\begin{gathered}
a_{1 t t}^{0}(r)=\chi_{1}\left[\left(a_{1 r r}^{0}+\frac{1}{r} a_{1 r}^{0}\right)_{r r}+\frac{1}{r}\left(a_{1 r r}^{0}+\frac{1}{r} a_{1 r}^{0}\right)_{r}\right], \\
\bar{a}_{2 t t}^{0}(r)=\chi_{2}\left[\left(a_{2 r r}^{0}+\frac{1}{r} a_{2 r}^{0}\right)_{r r}+\frac{1}{r}\left(a_{2 r r}^{0}+\frac{1}{r} a_{2 r}^{0}\right)_{r}\right]-\frac{\alpha^{\prime \prime}(0)\left(r-R_{1}\right)^{2}}{\left(R_{2}-R_{1}\right)^{2}} .
\end{gathered}
$$

Further we have

$$
\begin{gathered}
A_{5}(t)=k_{1} \int_{0}^{R_{1}} r\left(a_{1 t t}^{0}\right)^{2} d r+k_{2} \int_{R_{1}}^{R_{2}} r\left(\bar{a}_{2 t t}^{0}\right)^{2} d r+ \\
+\frac{\rho_{2} c_{\rho_{2}}}{\left(R_{2}-R_{1}\right)^{2}} \int_{0}^{t} \int_{R_{1}}^{R_{2}} r\left[2 \chi_{2} \alpha^{\prime \prime}(\tau)\left(2-\frac{R_{1}}{r}\right)-\alpha^{\prime \prime \prime}(\tau)\left(r-R_{1}\right)^{2}\right] d r .
\end{gathered}
$$

Similarly to function $A(t)$ the function $A_{4}(t)$ satisfies an estimate of type (15), and hence (21) with the replacement $A_{0}$ by $A_{40}, \alpha(t)$ by $\alpha^{\prime \prime}(\tau)$ and $\alpha^{\prime}(\tau)$ by $\alpha^{\prime \prime \prime}(\tau)$.

If we require convergence of the integral

$$
\begin{gathered}
\int_{0}^{\infty}\left|\alpha^{\prime \prime \prime}(\tau)\right| e^{\eta t} d \tau<\infty \\
\left|\alpha^{\prime \prime \prime}(t)\right|=\alpha_{4}(t) e^{-\eta t}, \quad \int_{0}^{\infty} \alpha_{4}(\tau) d \tau<\infty,
\end{gathered}
$$


we obtain an estimate of the function $A_{5}(t)$ (we use the formula (22)

$$
\begin{gathered}
\left|A_{5}(t)\right| \leqslant k_{1} \int_{0}^{R_{1}} r\left(a_{1 t t}^{0}\right)^{2} d r+k_{2} \int_{R_{1}}^{R_{2}} r\left(\bar{a}_{2 t t}^{0}\right)^{2} d r+ \\
+\rho_{2} c_{\rho_{2}} R_{2}\left[\frac{4 \chi_{2}}{R_{2}-R_{1}} \int_{0}^{t}\left|\alpha^{\prime \prime}(\tau)\right| d \tau+\left(R_{2}-R_{1}\right) \int_{0}^{t}\left|\alpha^{\prime \prime \prime}(\tau)\right| d \tau\right],
\end{gathered}
$$

where $a_{j t t}^{0}(r)$ are defined by formulas (24). By virtue of (41), (49) $\left|A_{5}(t)\right| \leqslant A_{5}(\infty)$ and, similarly to estimate (21), we obtain from (45)

$$
\begin{gathered}
\left|a_{2 t t}(r, t)\right| \leqslant \alpha_{4}(t) e^{-\eta t}+2\left(\frac{A_{5}(\infty) D_{2}^{2}}{R_{1}^{2} k_{2} \rho_{2} c_{\rho_{2}}}\right)^{1 / 4} e^{-\eta t / 2} \\
D_{2}=\sqrt{A_{40}}+\left[\max _{j}\left(\frac{1}{\rho_{j} c_{\rho_{j}}}\right)\right]^{1 / 2}\left[\frac{4 \chi_{2}}{\left(R_{2}-R_{1}\right)^{3 / 2}} \int_{0}^{\infty}\left|\alpha^{\prime \prime}(\tau)\right| e^{\eta \tau} d \tau+\right. \\
\left.+\sqrt{R_{2}\left(R_{2}-R_{1}\right)} \int_{0}^{\infty}\left|\alpha^{\prime \prime \prime}(\tau)\right| e^{\eta \tau} d \tau\right]
\end{gathered}
$$

We proceed to elaboration the estimates of the functions $v_{j}(r, t), f_{j}(t)$, when $\alpha(\tau), \alpha^{\prime}(\tau)$, $\alpha^{\prime \prime}(\tau)$ and $\alpha^{\prime \prime \prime}(\tau)$ satisfy conditions $(23)-(25),(41),(42)$. In this case everywhere we replace $a_{1}\left(R_{1}, t\right), a_{1 t}\left(R_{1}, t\right)$ by $a_{2}\left(R_{1}, t\right), a_{2 t}\left(R_{1}, t\right)$ according to the first equation (11). We begin with the function $v_{2}(r, t)$, for which inequality $(28)$ is proved. The quantity $E(t)$ entering the righthand side of this inequality has estimate (34), where $H_{1}(t)$ is given by (36) than from (37) we obtain

$$
\begin{gathered}
\int_{R_{1}}^{R_{2}} r Q_{2}^{2}(r, t) d r \leqslant \frac{8 æ^{2}}{\mu_{2}^{2}}\left[a_{2 t}^{2}\left(R_{1}, t\right) \int_{R_{1}}^{R_{2}} r P_{4}^{2}(r) d r+\nu_{1}^{2} a_{2}^{2}\left(R_{1}, t\right) \int_{R_{1}}^{R_{2}} r\left(P_{4 r r}+\frac{1}{r} P_{4 r}\right)^{2} d r\right] \equiv \\
\equiv d_{3} a_{2}^{2}\left(R_{1}, t\right)+d_{4} a_{2 t}^{2}\left(R_{1}, t\right) .
\end{gathered}
$$

So the inequality is valid

$$
\begin{aligned}
H_{1}(t) \leqslant & \frac{æ}{\sqrt{\rho_{1}}}\left|a_{2}\left(R_{1}, t\right)\right|+\sqrt{\frac{\rho_{2}}{2}}\left(\sqrt{d_{3}}\left|a_{2}\left(R_{1}, t\right)\right|+\sqrt{d_{4}}\left|a_{2 t}\left(R_{1}, t\right)\right|\right)= \\
& =\left(\frac{\Re}{\sqrt{\rho_{1}}}+\sqrt{\frac{\rho_{2} d_{3}}{2}}\right)\left|a_{2}\left(R_{1}, t\right)\right|+\sqrt{\frac{\rho_{2} d_{4}}{2}}\left|a_{2 t}\left(R_{1}, t\right)\right|
\end{aligned}
$$

and estimate (34) takes the form

$$
\begin{gathered}
E(t) \leqslant\left[\sqrt{E(0)}+\left(\frac{\Re}{\sqrt{\rho_{1}}}+\sqrt{\frac{\rho_{2} d_{3}}{2}}\right) \int_{0}^{t}\left|a_{2}\left(R_{1}, t\right)\right| e^{\delta \tau} d \tau+\right. \\
\left.+\sqrt{\frac{\rho_{2} d_{4}}{2}} \int_{0}^{t}\left|a_{2 t}\left(R_{1}, t\right)\right| e^{\delta \tau} d \tau\right]^{2} e^{-2 \delta t} .
\end{gathered}
$$

According to estimates (26), (43) the integrals in (54) have the order $e^{(\delta-\eta) t}$ and $e^{(\delta-\eta / 2) t}$ for large $t$, therefore we obtain

$$
E(t) \leqslant \gamma(t), \quad \text { where } \quad \gamma(t) \equiv d_{5} \begin{cases}e^{-2 \delta t}, & \delta<\eta / 2 \\ t e^{-2 \delta t}, & \delta=\eta / 2 \\ e^{-\eta t}, & \delta>\eta / 2\end{cases}
$$


with positive constant $d_{5}$.

Defined by equality (38) with using (53) the function $H_{2}(t)$ is evaluated as follows:

$$
\begin{aligned}
H_{2}(t) \leqslant & \mu_{2} \int_{R_{1}}^{R_{2}} r\left(\bar{v}_{2 r}^{0}\right)^{2} d r+\left(\frac{\rho_{2} d_{3}}{2}+\frac{\Re^{2}}{\rho_{1}}\right) \int_{0}^{t} a_{2}^{2}\left(R_{1}, \tau\right) d \tau+ \\
& +\frac{\rho_{2} d_{4}}{2} \int_{0}^{t} a_{2 \tau}^{2}\left(R_{1}, \tau\right) d \tau \leqslant D_{2}=\text { const }>0
\end{aligned}
$$

by virtue of inequalities (26), (43).

So from (13), (54), (55) we find estimate

$$
\begin{aligned}
&\left|v_{2}(r, t)\right| \leqslant \frac{2 æ}{\mu_{2}} \max _{r \in\left[R_{1}, R_{2}\right]} \mid P_{4}(r) \mid\left[\alpha_{1}(t) e^{-\eta t}+2\left(\frac{A_{1}(\infty) D^{2}}{R_{1}^{2} k_{2} \rho_{2} c_{\rho_{2}}}\right)^{1 / 4} e^{-\eta t / 2}\right]+ \\
&+\sqrt{2}\left(\frac{2 d_{5}}{R_{1}^{2} \nu_{2}} D_{2} \gamma(t)\right)^{1 / 4}
\end{aligned}
$$

and $v_{2}(r, t)$ approaches to zero uniformly over $r \in\left[R_{1}, R_{2}\right]$ with increasing time $t$.

Below we need the values $f_{j}(0)$. From $(7)$ we obtain the connection between them

$$
\rho_{1} f_{1}(0)=\rho_{2} f_{2}(0)-\frac{2 æ}{R_{1}} a_{1}^{0}\left(R_{1}\right) .
$$

The other relation follows from the second equality (3) and equation (5) (we recall that $\left.v_{j}(r, 0)=0\right)$ :

Now we find

$$
f_{1}(0)=-\frac{R_{2}^{2}-R_{1}^{2}}{R_{1}^{2}} f_{2}(0)
$$

$$
f_{1}(0)=\frac{2 æ\left(R_{2}^{2}-R_{1}^{2}\right) a_{1}^{0}\left(R_{1}\right)}{R_{1}^{2}+\rho\left(R_{2}^{2}-R_{1}^{2}\right)}, \quad f_{2}(0)=\frac{R_{1}^{2}}{R_{2}^{2}-R_{1}^{2}} f_{1}(0) .
$$

Moreover the relations are valid

$$
v_{1 t}(r, 0)=f_{1}(0), \quad \bar{v}_{2 t}(r, 0)=f_{2}(0)+\frac{2 æ \chi_{1}}{\mu_{2}}\left(a_{1 r r r}^{0}+\frac{1}{r} a_{1 r r}^{0}\right) P_{4}(r) .
$$

The second initial condition follows from the equations

$$
\left|a_{1}\left(R_{1}, t\right)\right|=\left|a_{2}\left(R_{1}, t\right)\right| \leqslant|\alpha(t)|+2\left(\frac{1}{R_{1}^{2} k_{2} \rho_{2} c_{\rho_{2}}} A(t) A_{1}(t)\right)^{1 / 4},
$$

and (37) and replacement

$$
v_{2}(r, t)=\bar{v}_{2}(r, t)-\frac{2 æ a_{1}\left(R_{1}, t\right)}{\mu_{2}} P_{4}(r) .
$$

We consider the following inequality that was obtained in [1]

$$
\left|v_{2 t}(r, t)\right| \leqslant \frac{2 æ}{\mu_{2}}\left|a_{1 t}\left(R_{1}, t\right)\right| \max _{r \in\left[R_{1}, R_{2}\right]}\left|P_{4}(r)\right|+\sqrt{\frac{2}{R_{1}}}\left(\frac{2}{\rho_{2} \mu_{2}} H_{3}(t) E_{1}(t)\right)^{1 / 4} .
$$

The function $E_{1}(t)$ on the right-hand side of inequality (61) has the form

$$
E_{1}(t)=\frac{\rho_{1}}{2} \int_{0}^{R_{1}} r v_{1 t}^{2} d r+\frac{\rho_{2}}{2} \int_{R_{1}}^{R_{2}} r \bar{v}_{2 t}^{2} d r
$$




$$
E_{1}(0)=\frac{\rho_{1} R_{1}^{2}}{4} f_{1}^{2}(0)+\frac{\rho_{2}}{2} \int_{R_{1}}^{R_{2}} r \bar{v}_{2 t}^{2}(r, 0) d r
$$

where $f_{1}(0)$ and $\bar{v}_{2 t}(r, 0)$ are defined by the first (57) and the second (58) equality respectively. There is the estimate form (54) for $E_{1}(t)$.

$$
\begin{aligned}
E_{1}(t) \leqslant\left[\sqrt{E_{1}(0)}+\left(\frac{æ}{\sqrt{\rho_{1}}}+\sqrt{\frac{\rho_{2} d_{3}}{2}}\right) \int_{0}^{t}\left|a_{2 t}\left(R_{1}, \tau\right)\right| e^{\delta \tau} d \tau+\right. \\
\left.\quad+\sqrt{\frac{\rho_{2} d_{4}}{2}} \int_{0}^{t}\left|a_{2 t t}\left(R_{1}, \tau\right)\right| e^{\delta \tau} d \tau\right]^{2} e^{-2 \delta t}
\end{aligned}
$$

Taking into account the obtained estimates (43), (51) from (53) we find using the constant $d_{6}$ the inequality

$$
E_{1}(t) \leqslant d_{6} \gamma(t)
$$

and the function $\gamma(t)$ from inequality (55).

For the function $H_{3}(t)$, from the right-hand side of inequality (61) we have the expression

$$
\begin{aligned}
H_{3}(t)=\mu_{1} \int_{0}^{R_{1}} r\left(v_{1 t r}^{0}\right)^{2} d r+\mu_{2} & \int_{R_{1}}^{R_{2}} r\left(\bar{v}_{2 t r}^{0}\right)^{2} d r+ \\
& +\frac{\rho_{2}}{2} \int_{0}^{t} \int_{R_{1}}^{R_{2}} r Q_{3}^{2}(r, \tau) d r d \tau+\frac{æ^{2}}{\rho_{1}} \int_{0}^{t} a_{2}^{2}\left(R_{1}, \tau\right) d \tau,
\end{aligned}
$$

where in our case

$$
\begin{gathered}
Q_{3}(r, t)=\frac{2 æ}{\mu_{2}}\left[-\nu_{2} a_{2 t}\left(R_{1}, t\right)\left(P_{4 r r}+\frac{1}{r} P_{4 r}\right)+a_{2 t t}\left(R_{1}, t\right) P_{4}(r)\right], \\
v_{1 t r}^{0}(r)=0, \quad \bar{v}_{2 t r}^{0}=\frac{2 æ}{\mu_{2}} a_{2 t}\left(R_{1}, 0\right) P_{4 r}, \\
a_{2 t}\left(R_{1}, 0\right)=\chi_{2}\left[a_{2 r r}^{0}\left(R_{1}\right)+\frac{1}{R_{1}} a_{2 r}^{0}\left(R_{1}\right)\right] .
\end{gathered}
$$

It is clear that

$$
\int_{R_{1}}^{R_{2}} r Q_{3}^{2}(r, t) d r \leqslant d_{3} a_{2 t}^{2}\left(R_{1}, t\right)+d_{4} a_{2 t t}^{2}\left(R_{1}, t\right)
$$

with constant $d_{3}, d_{4}$ from (52). By virtue of the convergence of the integrals

$$
\int_{0}^{\infty}\left(a_{2}^{(k)}(\tau)\right)^{2} d \tau, \quad k=0,1,2
$$

we obtain the inequality $H_{3}(t) \leqslant H_{3}(\infty)$ and estimate (61) takes the form for all $r \in\left[R_{1}, R_{2}\right]$

$$
\begin{aligned}
\left|v_{2 t}(r, t)\right| \leqslant \frac{2 æ}{\mu_{2}} \max _{r \in\left[R_{1}, R_{2}\right]}\left|P_{4}(r)\right|\left[\alpha_{2}(t) e^{-\eta t}+2\left(\frac{A_{3}(\infty) D_{1}^{2}}{R_{1}^{2} k_{2} \rho_{2} c_{\rho_{2}}}\right)^{1 / 4} e^{-\eta t / 2}\right]+ \\
+\sqrt{2}\left(\frac{2 d_{6}}{R_{1}^{2} \nu_{2}} H_{3}(\infty) \gamma(t)\right)^{1 / 4} .
\end{aligned}
$$

The function $f_{1}(t)$ is the pressure gradient in the first fluid along the axis $z$. The function $g(t)$ on the right side of the inequality (29) has form

$$
g(t)=R_{1}^{2} v_{2}\left(R_{1}, t\right)+2 \int_{R_{1}}^{R_{2}} r v_{2}(r, t) d r
$$


and, taking into account estimate (56), we find

$$
\begin{aligned}
|g(t)| \leqslant R_{2}^{2}\left\{\frac { 2 æ } { \mu _ { 2 } } \operatorname { m a x } _ { r \in [ R _ { 1 } , R _ { 2 } ] } | P _ { 4 } ( r ) | \left[\alpha_{1}(t) e^{-\eta t}\right.\right. & \left.+2\left(\frac{A_{1}(\infty) D^{2}}{R_{1}^{2} k_{2} \rho_{2} c_{\rho_{2}}}\right)^{1 / 4} e^{-\eta t / 2}\right]+ \\
& \left.+\sqrt{2}\left(\frac{2 d_{5}}{R_{1}^{2} \nu_{2}} H_{2}(\infty) \gamma(t)\right)^{1 / 4}\right\} \leqslant d_{7} e^{-\omega t}
\end{aligned}
$$

where $\omega=\min (\delta / 2, \eta / 4)$ (at $\delta=\eta / 2$ in (66) there is $t e^{-\omega t}$ instead of $e^{-\omega t}$ according to (54)).

Now from (29) using inequalities (65), (66) we obtain the estimate

$$
\begin{aligned}
& \left|f_{1}(t)\right| \leqslant 2 \nu_{1}\left[S_{1} d_{7} e^{-\omega t}+S_{2} d_{7}\left|\exp \left(-\frac{\zeta_{1}^{2} \nu_{1}}{R_{1}^{2}} t\right)-e^{-\omega t}\right|\right]+d_{8} e^{-\omega t}, \\
& S_{1}=\frac{1}{7} R_{1}^{4}+\sum_{n=1}^{\infty}\left|h_{n}^{2}\right|, \quad S_{2}=\nu_{1} \sum_{n=1}^{\infty} \frac{1}{\nu_{1} R_{1}^{-2} \zeta_{n}^{2}-\omega}\left(\left|h_{n}^{1}\right|+\frac{\zeta_{n}^{2}}{R_{1}^{2}}\left|h_{n}^{2}\right|\right),
\end{aligned}
$$

at that $S_{1}<\infty$ and $S_{2}<\infty$. The estimate $f_{2}(t)$ follows from (5), inequalities (26) and (67)

$$
\left|f_{2}(t)\right| \leqslant \rho\left|f_{1}(t)\right|+2 æ\left[\alpha_{1}(t) e^{-\eta t}+2\left(\frac{A_{1}(\infty) D^{2}}{R_{1}^{2} k_{2} \rho_{2} c_{\rho_{2}}}\right)^{1 / 4} e^{-\eta t / 2}\right] .
$$

Remark 1. From inequality (30), estimates (56) and (67) it follows that the function $v_{1}(r, t)$ tends exponentially to zero with increasing time.

$$
\begin{aligned}
\left|v_{1}(r, t)\right| \leqslant R_{1} \max _{t \in[0, T]}\left|\frac{2 a}{\mu_{2}} \max _{r \in\left[R_{1}, R_{2}\right]}\right| P_{4}(r) \mid\left[\alpha_{1}(t) e^{-\eta t}+2\left(\frac{A_{1}(\infty) D^{2}}{R_{1}^{2} k_{2} \rho_{2} c_{\rho_{2}}}\right)^{1 / 4} e^{-\eta t / 2}\right]+ \\
+\sqrt{2}\left(\frac{2 d_{5}}{R_{1}^{2} \nu_{2}} D_{2} \gamma(t)\right)^{1 / 4}\left|+\frac{2 R_{1}}{\nu_{1}} \max _{t \in[0, T]}\right| 2 \nu_{1}\left[S_{1} d_{7} e^{-\omega t}+\right. \\
\left.+S_{2} d_{7}\left|\exp \left(-\frac{\zeta_{1}^{2} \nu_{1}}{R_{1}^{2}} t\right)-e^{-\omega t}\right|\right]+d_{8} e^{-\omega t} \mid \sum_{n=1}^{\infty} \frac{1}{\xi_{n}^{3}\left|J_{1}\left(\xi_{n}\right)\right|} .
\end{aligned}
$$

For the function $h_{1}(t)$ from (12), taking into account the first relation (3) and the inequality (56) we have the estimate

$$
\begin{gathered}
\left|h_{1}(t)\right| \leqslant \frac{R_{2}^{2}-R_{1}^{2}}{2 R_{1}}\left\{\frac { 2 æ } { \mu _ { 2 } } \operatorname { m a x } _ { r \in [ R _ { 1 } , R _ { 2 } ] } | P _ { 4 } ( r ) | \left[\int_{0}^{t} \alpha_{1}(\tau) e^{-\eta \tau} d \tau+\right.\right. \\
\left.\left.+\frac{4}{\eta}\left(\frac{A_{1}(\infty) D^{2}}{R_{1}^{2} k_{2} \rho_{2} c_{\rho_{2}}}\right)^{1 / 4}\left(1-e^{-\eta t / 2}\right)\right]+\sqrt{2}\left(\frac{2 d_{5}}{R_{1}^{2} \nu_{2}} H_{2}(\infty)\right)^{1 / 4} \int_{0}^{t} \gamma^{1 / 4}(\tau) d \tau\right\}
\end{gathered}
$$

and $h_{1}(t)$ is limited at $t \rightarrow \infty$.

Thus, it is proofed

Theorem 2.1. If the function $\alpha(\tau), \alpha^{\prime}(\tau), \alpha^{\prime \prime}(\tau), \alpha^{\prime \prime \prime}(\tau)$ satisfy conditions (23)- $(25)$, (41), (42), (49), then the following estimates (26), (27), (56), (67), (68), (69) are valid for the functions $a_{j}(r, t), v_{j}(r, t), f_{j}(t)$ from which it follows that these functions tend exponentially to zero with increasing time.

Remark 2. Remark 6. Conditions (23)-(25), (41), (42), (49) physically mean that the thermal effects on the solid wall surface of cylinder at $r=R_{2}$ are very small and the braking of liquids occurs at $t \rightarrow \infty$ due to frictional forces. 


\section{References}

[1] V.K.Andreev, E.P.Magdenko, Journal of Siberian Federal University. Mathematics \& Physics, 12(2019), no. 4, 1-13, DOI: 10.17516/1997-1397-2019-12-4-483-495.

[2] G.Bateman, A.Erdein, Higher transcendental functions. Bessel functions, parabolic cylinder functions, orthogonal polynomials, Moscow, Nauka, 1974 (in Russian); McGraw-Hill, 1953.

[3] A.P.Prudnikov, Y.A.Bychkov, O.I.Marichev, Integrals and series. Special functions, Moscow, Nauka, 1983 (in Russian).

\section{Об асимптотическом поведении сопряженной задачи, описывающей ползущее осесимметричное термокапиллярное движение}

Виктор К. Андреев

Евгений П. Магденко

Институт вычислительного моделирования СО РАН

Красноярск, Российская Федерация

Сибирский федеральный университет

Красноярск, Российская Федерация

Аннотация. В работе указаны условия для закона поведения температуры на твердой стенке цилиндра, при которых решение линейной сопряженной обратной начально-краевой задачи, описывающей двухслойное осесимметрическое ползущее движение вязких теплопроводных жидкостей, с ростом времени экспоненциально стремится к нулю.

Ключевые слова: сопряженная нелинейная обратная задача, поверхность раздела, ползущее движение. 